\title{
Control of replication initiation and heterochromatin formation in Saccharomyces cerevisiae by a regulator of meiotic gene expression
}

\author{
Horst Irlbacher, ${ }^{1,3}$ Jacqueline Franke, ${ }^{1,3}$ Thomas Manke, ${ }^{2}$ Martin Vingron, ${ }^{2}$ and \\ Ann E. Ehrenhofer-Murray ${ }^{1,3,4}$ \\ ${ }^{1}$ Otto-Warburg-Laboratorium and ${ }^{2}$ Department for Computational Molecular Biology, Max-Planck-Institut für Molekulare \\ Genetik, D-14195 Berlin, Germany; ${ }^{3}$ Institut für Genetik, Justus-Liebig-Universität Giessen, D-35392 Giessen, Germany
}

\begin{abstract}
Heterochromatinization at the silent mating-type loci $H M R$ and $H M L$ in Saccharomyces cerevisiae is achieved by targeting the Sir complex to these regions via a set of anchor proteins that bind to the silencers. Here, we have identified a novel heterochromatin-targeting factor for $H M L$, the protein Sum 1 , a repressor of meiotic genes during vegetative growth. Sum 1 bound both in vitro and in vivo to $H M L$ via a functional element within the $H M L$-E silencer, and sum1 $1 \Delta$ caused $H M L$ derepression. Significantly, Sum 1 was also required for origin activity of $H M L-E$, demonstrating a role of Sum1 in replication initiation. In a genome-wide search for Sum1-regulated origins, we identified a set of autonomous replicative sequences (ARS elements) that bound both the origin recognition complex and Sum1. Full initiation activity of these origins required Sum1, and their origin activity was decreased upon removal of the Sum1-binding site. Thus, Sum1 constitutes a novel global regulator of replication initiation in yeast.
\end{abstract}

[Keywords: HML-D; Hst1; chromatin; replication; origin; ARS]

Supplemental material is available at http://www.genesdev.org.

Received December 17, 2004; revised version accepted June 1, 2005.

The division of eukaryotic chromatin into functionally distinct domains is critical to implement gene expression programs that drive the development of multicellular organisms. Regions termed euchromatin exist in the genome that are generally conducive to transcription, whereas heterochromatin contains specialized chromatin-binding proteins that repress transcription in these regions. A central question in heterochromatin biology is how the heterochromatin factors are targeted to specific genomic regions, a process that is crucial to ensure that the designated domains, and only they, are repressed in the appropriate spatial and temporal fashion. While replication initiation factors have been invoked in this process, little is known about its regulation during development.

In the yeast Saccharomyces cerevisiae, heterochromatin-like structures are targeted via a set of DNA-binding factors to the silent mating-type loci $H M L \alpha$ and $H M R \boldsymbol{a}$, the telomeres, and the rDNA locus (Rusche et al. 2003). For $H M$ and telomeric silencing, these factors include

${ }^{4}$ Corresponding author.

E-MAIL ehrenhof@molgen.mpg.de; FAX 49-30-8413-1130.

Article and publication are at http://www.genesdev.org/cgi/doi/10.1101/ gad.334805. the proteins Rap1 and Abf1, which function as transcriptional activators elsewhere in the genome /Shore and Nasmyth 1987; Kimmerly et al. 1988; Halfter et al. 1989), and the origin recognition complex ORC (Bell et al. 1993; Foss et al. 1993), the replication initiator that is conserved throughout eukaryotes. At the $H M$ loci, the binding sites for these recruitment factors are confined to the so-called silencers E and I that flank the repressed regions. All four $H M$ silencers contain a binding site for ORC; $H M R$-E and $H M L$-E contain a Rap1-binding site; and $H M R$-E, $H M R$-I, and $H M L-I$ encompass an Abf1binding site (Rusche et al. 2003).

While much effort has concentrated on analysis of the $H M R$ silencers, one functional domain within the $H M L$-E silencer has escaped molecular characterization. The HML-E silencer requires a region termed the $\mathrm{D}$ element for full silencing activity (Mahoney et al. 1991). This 93-bp sequence was identified more than a decade ago by deletion analysis, but contains no binding motifs for any of the known targeting factors. Thus, the function of $H M L-\mathrm{D}$ so far has remained elusive.

Interestingly, the silencers all have the ability to confer replication initiation to plasmids that lack an origin, a feature that is termed ARS (autonomous replicative sequence) activity. However, only $H M R$-E and $H M R$-I 
are origins of replication at their native chromosomal locations (Rivier and Rine 1992; Rivier et al. 1999), whereas the $H M L$ silencers are inactivated by replication forks initiating at early-firing origins in the vicinity (Sharma et al. 2001), and hence are not active in the chromosome. In line with this, replication initiation by ORC at the silencers is dispensable for silencing (Ehrenhofer-Murray et al. 1995). Rather, ORC function in silencing requires that the Orcl subunit physically contact Sir1 (Triolo and Sternglanz 1996), which together with Rap1 interacts with the structural components of yeast heterochromatin, Sir2, Sir3, and Sir4. Sir2 is a $\mathrm{NAD}^{+}$-dependent histone deacetylase (HDAC) that deacetylates the N-terminal tails of histones $\mathrm{H} 3$ and $\mathrm{H} 4$ (Imai et al. 2000), thereby establishing the chromatin modification state necessary for Sir3 and Sir4 binding (Hecht et al. 1995). Thus, the Sir2/3/4 complex polymerizes across the chromosome to create silent chromatin (Hoppe et al. 2002; Rusche et al. 2002). Significantly, the heterochromatin-targeting function of ORC is conserved in metazoans. Drosophila ORC1 interacts with heterochromatin protein 1 (HP-1), and mutations in DmORC2 cause a loss of position effect variegation, a form of heterochromatin-mediated silencing in flies (Pak et al. 1997).

Heterochromatin shares several features with genespecific repression, a locally confined process in which transcriptional repressors bind to sites upstream of the gene to be repressed and recruit HDACs to alter histone acetylation patterns. One such example is the Sum 1 protein, which acts as a repressor of a subset of meiotic genes during the mitotic cell cycle (Xie et al. 1999). In this function, Sum1 recruits the Sir2 homolog Hst1 to deacetylate histones in the promoter region of some, but not all of the Sum1-repressed genes (McCord et al. 2003). Moreover, Sum 1 and Hst1 both interact with the tethering factor Rfm1 (repression factor of MSE [middle sporulation element]), thus targeting Hst1 to a subset of Sum1regulated genes (McCord et al. 2003). Intriguingly, Sum1 has been implicated in heterochromatin formation in an unusual context in that a dominant allele of SUM1, SUM1-1 (Livi et al. 1990), renders HM silencing dependent on the HDAC Hst1, but not Sir2 or the other Sir proteins (Rusche and Rine 2001; Sutton et al. 2001). The mutant phenotype is caused by a single amino acid change (Chi and Shore 1996; Rusche and Rine 2001) that improves the ability of the Sum 1 protein to interact with ORC at the $H M$ silencers, such that Sum1-1 recruits Hst1 instead of Sir2 to establish repression (Rusche and Rine 2001; Sutton et al. 2001). However, wild-type Sum1 so far has not been implicated in silencing.

Importantly, while ORC is the replication initiator, an ORC-binding site alone is not sufficient for replicator function. Rather, ARSs and origins contain multiple determinants that enhance initiation activity. All origins contain a perfect or close match to the ARS consensus sequence (ACS), the ORC-binding site. In addition, the ARS1 origin, perhaps the best-studied ARS sequence, contains three so-called B elements that stimulate autonomous replication (Marahrens and Stillman 1992).
The sequence closest to the ACS, B1, cooperates in ORC binding (Lee and Bell 1997); B2 is required for loading of the MCM complex (Zou and Stillman 2000; Wilmes and Bell 2002); and B3 is bound by Abf1 (Diffley and Stillman 1988). Abf1 is an accessory factor for origin function at a subset of chromosomal replication origins (Eisenberg et al. 1988; Rhode et al. 1992). Abf1 sites are found in several origins, and in three of the four HM silencers (Kimmerly et al. 1988). Also, Mcm1-binding sites at a distance from the ACS influence initiation at subtelomeric sequences (Chang et al. 2004). Notably, there is little sequence conservation between individual ARSs outside of the ACS, supporting the notion that there are many ways that cells can assemble functional origins, and that many of these modular origin determinants remain to be characterized.

In this study, we undertook a molecular characterization of the D element within $H M L$-E. This led us to the discovery that the Sum 1 protein bound $H M L-\mathrm{D}$ in vitro and in vivo and was required for full $H M L$ repression. Thus, Sum 1 acts not only in local, gene-specific repression at early-meiotic genes, but also was important for the creation of more extensive silenced domains. In addition, we uncovered a role for Sum 1 as a modulator of replication initiation at $H M L-\mathrm{E}$ as well as a subset of other chromosomal origins. In line with this, we found that sum1s was synthetically lethal with orc2-1 and orc5-1 and caused synthetic growth defects in cdc6-1, cdc7-1, and cdc45-1 strains. These findings underscore the variability of auxiliary determinants at replication origins and in heterochromatin recruitment and provide insight into the regulation of both origin usage and the creation of silent domains under varying conditions.

\section{Results}

Defining a core region within the $D$ element of HML-E

Previous deletion analysis of the $H M L$-E silencer defined three functional elements within a $150-b p$ region that are essential for silencer function: a Rap1-binding site (RAP), an ORC-binding site (ARS consensus sequence, ACS), and a third region termed the D element (Fig. 1A; Mahoney et al. 1991). These elements were defined by deletions in the $H M L$-E silencer in a strain lacking $H M L$ I. When removed individually, they resulted in little derepression, whereas the deletion of any two elements caused a severe loss of silencing (Fig. 1B; Mahoney et al. 1991). The D element, as it is currently outlined, spans $93 \mathrm{bp}$ and starts 6 bp centromere-proximal to the ACS. It thus represents a relatively large, unmapped DNA segment with no assigned function. Since all other $H M$ silencer elements are binding sites for proteins or protein complexes, we hypothesized that $H M L$-D also harbors a binding site for an as-yet-unidentified silencing factor or complex, which we sought to identify in this study.

Factor-binding sites usually comprise 15-20 bp of DNA sequence. In a first set of experiments, we therefore asked whether $\mathrm{D}$ function could be narrowed down 
A
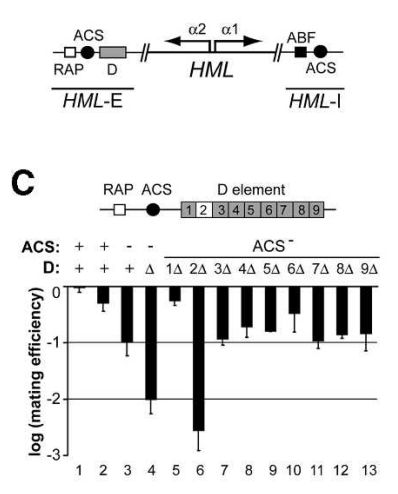

B

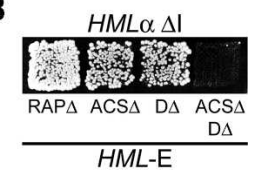

D

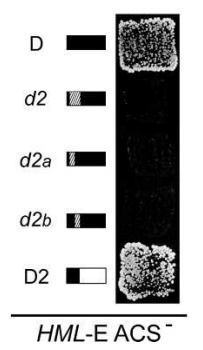

Figure 1. Identification of a D-element core region within the $H M L$-E silencer. $(A)$ Schematic representation of the $H M L \alpha$ locus on the left arm of chromosome III. Location and elements of the silencers $H M L$-E and $H M L$-I are indicated. (RAP) Rap1-binding site; (ACS) ORC-binding site; (D) D element; (ABF) ABFbinding site. $(B)$ Redundancy of $H M L$-E silencer elements. Loss of $H M L \alpha$ silencing in $H M L$-E silencer deletion mutants was measured as loss of a-mating ability in a patch mating assay. All strains were $H M L-\Delta \mathrm{I}$. (C) D2 was the core element of D. Quantitative mating assays were performed to compare the effect on silencing of different D-element deletions in a MATa $H M L$-E $\mathrm{ACS}^{-} \Delta \mathrm{I}$ strain background (AEY3395). The mean values of at least three independent experiments are shown. $(D)$ D2 was both necessary and sufficient for $H M L$-E function. Loss of silencing in $H M L$-E $\mathrm{ACS}^{-} \Delta \mathrm{I}$ strains was measured for the 14-bp sequence element containing the D2 element (D, AEY3395), mutations in the entire (d2, AEY3426), the first (d2a, AEY3430) or the second ( $d 2 b, \mathrm{AEY} 3434)$ half of the D2 element, or with the $\mathrm{D} 2$ element remaining as the sole $\mathrm{D}$ sequence at $H M L-\mathrm{E}$ (D2, AEY3552).

to a shorter DNA segment. This was achieved by systematically introducing 6-12-bp deletions across D and determining whether they caused $H M L \alpha$ derepression in a $M A T$ a strain lacking $H M L-I$ and carrying a mutation in the ACS of HML-E. Silencing in these strains was evaluated by determining their mating ability, because derepression of $H M L \alpha$ in MATa strains results in coexpression of a and $\alpha$ information, thus causing a nonmating phenotype. As in the original study (Mahoney et al. 1991), deletion of a sequence essential for $\mathrm{D}$ function in this experimental setup should lead to the same full derepression as deletion of the complete D element. Significantly, we found that a 10-bp deletion termed D2 that lies $16 \mathrm{bp}$ centromere-proximal to the ACS (position 133-143, numbering system based on Feldman et al. 1984), was sufficient to mimick the effect of a full Delement deletion. Removing other areas of $\mathrm{D}$ had no effect on $H M L$ silencing (Fig. 1C). These results suggested that sequences essential for D function were located within the D2 fragment.

We next asked whether D2 function could be abrogated by mutating rather than deleting the sequence, because a sequence deletion might not just remove a protein-binding site, but could alter silencing by other means, for instance, by changing nucleosome position

and chromatin architecture. Therefore, we mutated every other base pair by transition in a 14-bp region that contained D2, thus maintaining the purine/pyrimidine composition of the original area. We found that the fully mutated 14-bp area (termed d2) caused HML derepression just like the D2 deletion did (Fig. 1D). We used the same strategy to individually mutate the first or the second seven bp of this region (termed $d 2 a$ and $d 2 b$ ). Both $d 2 a$ and $d 2 b$ lead to a complete derepression of $H M L \alpha$ (Fig. 1D), indicating that sequences necessary for D function were present in both elements.

We furthermore determined whether the D2 element was not only necessary, but also sufficient for silencing. To this end, we measured the $H M L \alpha$ silencing properties of a strain that had all of the D element removed except for the D2 sequence. We found that the D2 element alone was as efficient in $H M L \alpha$ silencing as the entire D element (Fig. 1D). Taken together, these results showed that the D2 region was the core sequence of the D element and that it was both necessary and sufficient for full D function. Furthermore, because this element is comparable in length to the Rap1- and ORC-binding sites, this suggested that $\mathrm{D} 2$ contained a binding site for a protein (complex) essential for silencing.

sum $1 \Delta$ caused $H M L$ derepression and was epistatic to the $D$ element

We next sought to genetically identify the hypothesized D-binding factor. One prediction for a mutation or deletion in the gene encoding this factor is that it causes derepression when silencing is compromised at $H M L$, but not $H M R$, because only $H M L$, but not $H M R$, contains a D element. More specifically, this mutation is expected to cause strong derepression only when $H M L$ silencing is weakened, for instance, by mutations in RAP or ACS of $H M L$-E in an $H M L-\Delta \mathrm{I}$ background. In short, removal of the D-binding factor is expected to have the same silencing phenotypes as mutation of its binding site in the HML-E silencer and should be epistatic to the binding site deletion.

In genetic crosses to characterize $H M L$ silencing, we observed that a deletion of SUM1 exactly matched the genetic predictions for the D-binding factor. Since Sum 1 is a DNA-binding protein that, albeit only in its mutant form, has been implicated in silencing, we hypothesized that Sum1 might be the D-binding factor. To address this, we tested whether sum $1 \Delta$ might cause $H M$ derepression when ORC function was compromised by a NAT1 deletion. Nat1 is a component of NatA, an Nterminal acetyltransferase that acetylates Orc1, which is required for ORC silencing function (Geissenhoner et al. 2004). We found that sum $1 \Delta$ caused strong derepression at $H M L$, but not HMR (Fig. 2A). This suggested that Sum 1 acted in a parallel pathway to ORC in $H M L$ silencing. We therefore investigated the effect of sum1 $1 \Delta$ in the presence of mutations at $H M L$. Significantly, sum $1 \Delta$ caused a strong loss of $H M L$ silencing when RAP or ACS of $H M L$-E was deleted (Fig. 2A). However, sum $1 \Delta$ did not generally weaken $H M L$ silencing, because it did not 
A

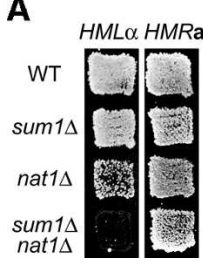

B

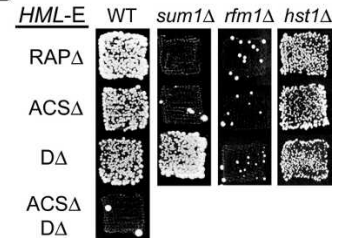

Figure 2. SUM1 was required for $H M L \alpha$ silencing and was epistatic to the D element. (A) Repression of $H M L \alpha$ or $H M R \mathbf{a}$ in strains deleted for SUM1, NAT1, or both was measured by patch mating assays. (B) SUM1, but not RFM1 or HST1, was genetically linked to $H M L-D$. $H M L \alpha$ silencing of sum $1 \Delta, r f m 1 \Delta$, or hst $1 \Delta$ strains in combination with silencer element deletions at $H M L$-E is shown by patch mating assays.

cause derepression when the $\mathrm{D}$ element was deleted. Thus, sum1D affected $H M L$ silencing as predicted for the D-binding factor in that it caused derepression when $H M L$ silencing was compromised and was epistatic to a deletion of the $\mathrm{D}$ element.

The Sum 1 protein has previously been characterized as a DNA-binding protein that functions as a repressor of a subset of meiotic genes during vegetative growth (Xie et al. 1999). A consensus Sum1-binding sequence has been defined (DSYGWCAYWDW) (Pierce et al. 2003), although this sequence is not found at all native Sum1binding sites and is also not present at HML-E. Normal Sum 1 has so far not been implicated in silencing, but the mutant Sum1-1 version (Laurenson and Rine 1991) is able to interact with ORC at the HMR-E silencer and recruits Rfm1 and Hst1 to establish a Sir-independent form of silencing (Chi and Shore 1996; Rusche and Rine 2001; Sutton et al. 2001; McCord et al. 2003). Intriguingly, Sum1-1 does not suppress sir $\Delta$ silencing defects at $H M L$ in some strain backgrounds (Laurenson and Rine 1991), whereas it does in others (Livi et al. 1990). Our results suggested that the normal Sum 1 protein acted in silencing by binding to a nonconsensus binding site within the D element of $H M L$-E. It is not surprising that a role for Suml at $H M L$ has so far not been observed (Chi and Shore 1996), because its effect is only apparent when $H M L$ silencing is weakened.

As Sum 1 in some instances recruits Rfm 1 and Hst 1 to repress transcription (McCord et al. 2003), we asked whether they were required like Sum 1 to silence $H M L$ fully. However, while deletion of RFM1 showed a general weakening of $H M L$ silencing at each of the single silencer deletion strains, the deletion of HST1 did not cause $H M L$ derepression (Fig. 2B). This indicated that Rfm1 had a role in $H M L$ silencing beyond Sum1, and that Sum1 did not cooperate with Hst1 in this context.

\section{Sum1 bound specifically to the D element} within HML-E

To test the notion that the Sum 1 protein was the Dbinding factor, we asked whether Sum 1 was able to bind $H M L$-E in vitro. To this end, we purified full-length
Sum1 (6xHis-tagged at the $\mathrm{N}$ terminus) from bacteria and performed electrophoretic mobility shift assays (EMSA) with $H M L$-E DNA. As a control, the purified 6xHis-Sum1 shifted DNA of a known Sum1-binding sequence, the SMK1 promoter (Xie et al. 1999), toward a slower mobility (Fig. 3D, lanes 1,2). Importantly, 6xHisSum1 also caused a 220-bp HML-E fragment to migrate more slowly (Fig. 3A, lanes 1,2), indicating that Sum1 bound to HML-E. This binding was competed away by adding unlabeled $H M L$-E DNA, but not by adding an unspecific 210-bp INO1 promoter region fragment, indicating specificity for $H M L$-E (Fig. 3A, lanes 3,4). Sum1 also did not bind the INO1 fragment in an individual binding assay (Fig. 3A, lanes 5,6). Also, the binding ability was unrelated to the 6xHis affinity tag, because $6 \times$ His-tagged $\beta$-galactosidase was unable to bind to $H M L$-E DNA (Fig. 3A, lanes 7,8).

To test whether the Sum1-mediated mobility shift of $H M L$-E DNA depended on the D element, we performed a series of EMSAs with mutated HML-E DNA. Whereas a shift was visible both with wild-type $H M L$-E and $H M L$-E with the ACS mutated, it was abolished when either the D element alone or the ACS and the D element together were mutated (Fig. 3B, lanes 1-8). This showed that Sum1 required the D element in order to bind to $H M L$-E. We also attempted EMSAs of Sum 1 with a 14-bp fragment containing the D2 element. However, Sum 1 was unable to bind to this short sequence (data not shown), indicating that neighboring sequences within the D element were necessary for full binding of Sum 1 in vitro.

To further test the involvement of D2 in Sum1 binding, we determined how the deletion of D2 affected the ability of $H M L$-E to bind Sum1. Whereas a 134-bp wildtype HML-E fragment bound Sum1 (Fig. 3C, lanes 1,2), binding was strongly decreased with a fragment of $H M L$-E lacking 10 bp of D2 (Fig. 3C, lanes 3,4). However, the binding was not as strongly reduced as with a complete deletion of the D element (Fig. 3C, lanes 5,6), indicating that sequences surrounding D2 influenced the binding affinity of Sum1.

Since Sum1 bound two unrelated sequences, SMK1 and $H M L-\mathrm{D}$, we were interested to determine whether the two sequences could compete with each other for Sum1 binding. Significantly, the mobility shift of Sum1 with SMK1 DNA was competed away by addition of a molar excess of $H M L$-E, but not by the same amount of $H M L$-E lacking the $\mathrm{D}$ element (Fig. 3D, lanes 2-4), thus showing a competition between the two fragments for Sum 1 binding. In summary, these experiments showed that Sum1 bound HML-E in vitro in a D2-dependent fashion.

\section{In vivo localization of Sum1 at HML-E}

We next asked whether Sum1 bound to HML-E in vivo. To this end, we performed chromatin immunoprecipitation (ChIP) experiments with 6xmyc-tagged Sum 1 . In the precipitates, we observed a weak, but consistent 2.5 -fold enrichment of $H M L$-E DNA in the presence of the $\alpha$-myc 
A

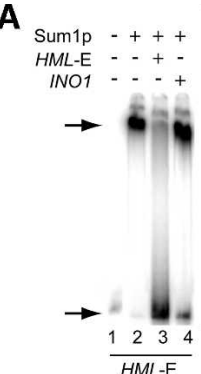

D Sum1p - +++ HML-E - - + HMLLE DS - -1

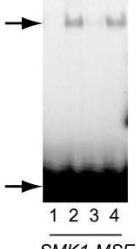

SMK1-MSE
B

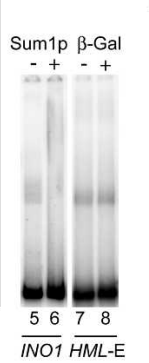

E

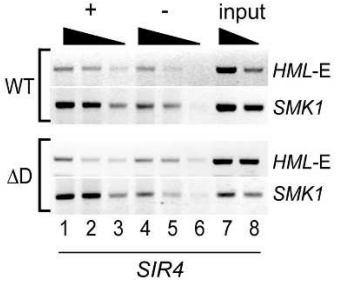

C

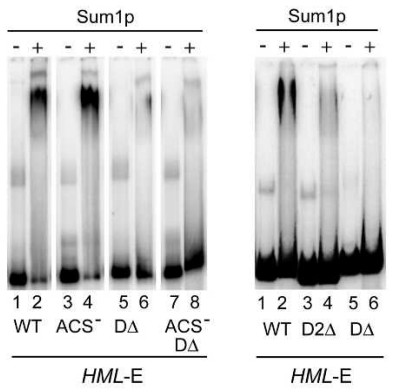

HML-E

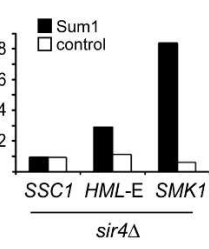

Figure 3. D-specific binding of Sum 1 to $H M L$-E in vitro and in vivo. (A) Sum 1 bound in vitro to $H M L$-E, but not the INO1 promoter region. (Left) A radioactively labeled 220-bp $H M L$-E fragment was incubated without protein (lane 1) or with $0.1 \mu \mathrm{M}$ bacterially expressed 6xHis-Sum1 (lanes 2-4). For competition experiments, unlabeled DNA of $H M L$-E (specific competitor, lane 3) or a 210-bp INO1 fragment (unspecific competitor, lane 4) was added. DNAprotein complexes were resolved on a polyacrylamide gel and labeled DNA was visualized by autoradiography. (Right) Sum1 did not bind INO1 DNA, and bacterially expressed 6xHis- $\beta$-galactosidase ( $\beta$-Gal) did not bind $H M L$-E DNA. (Upper arrow) Protein-DNA complex. (Lower arrow) Free DNA. (B) Binding of Sum1 to HML-E required the D element. Mutant versions of $H M L$-E were incubated with Sum $1(+)$ or without protein (-) and gel-electrophorezed as in $A$. $H M L$-E DNA containing a mutation in the ACS site is termed ACS (lanes 3,4,7,8), and $H M L$-E DNA with deletion of the 93-bp D element is termed D $\Delta$ (lanes 5-8). To maintain DNA size in the D $\Delta$ derivates, the deleted $\mathrm{D}$ element was substituted for the genomic 3 '-region of equivalent length. All DNA fragments were $\sim 220 \mathrm{bp} .(C)$ Binding of Sum 1 to $H M L$-E required the D2 element. Mutant versions of $H M L$-E were incubated with Sum $1(+)$ or without protein $(-)$ as in A. (WT) A 134-bp wild-type $H M L$-E fragment containing the ACS and the D element (lanes 1,2); (D2 $\Delta$ ) HML-E without the D2 element (lanes 3,4); (D $\Delta)$ a 140-bp HML-E fragment lacking the entire D element (lanes 5,6). (D) Competition between SMK1 and $H M L$-E for Sum1 binding. A radioactively labeled double-stranded 19-bp fragment containing the MSE site of the SMK1 promoter was incubated without protein (lane 1) or with $0.1 \mu M$ bacterially expressed 6xHis-Sum1 (lanes 2-4). For competition experiments, unlabeled DNA of $H M L$-E (specific competitor, lane 3) or $H M L$-E D $\Delta$ was added. (E) Sum 1 was associated in vivo with $H M L$-E in a D-element-dependent manner. ChIPs were performed on sum $1 \Delta$ strains containing a $2 \mu$ plasmid carrying $\mathrm{N}$-terminally 6xmyc-tagged SUM1 under control of its own promoter (pAE1032). (Left) (WT) wild-type HML $\alpha$ (AEY2); ( $\Delta \mathrm{D}) H M L \Delta \mathrm{D} \Delta \mathrm{I}(\mathrm{AEY} 3391)$. DNA was immunoprecipitated with (+) or without (-) anti-myc antibody and PCR-amplified. A total of $1 / 50$ or $1 / 100$ of the input DNA (lanes 7,8 ) or $1 / 2$ (lanes 1,4$), 1 / 4$ (lanes 2,5), or $1 / 8$ (lanes 3,6) of the immunoprecipitated DNA was analyzed. As a control, the promoter region of the SMK1 gene was PCR-amplified. (Right) ChIP was performed in sir4 $\Delta$ strains. Columns indicate the ratio of DNA enrichment with versus without anti-myc antibody: (black columns) 6xmyc Sum1; (white columns) untagged. The $Y$-axis indicates fold enrichment.

antibody as compared to ChIPs without antibody (Fig. 3E, left) or in strains lacking myc-tagged Sum1 (data not shown). In the same precipitates, the $S M K 1$ promoter, a known binding region for Sum1, was enriched eightfold (Fig. 3E), whereas the unrelated SSC1 gene promoter was not enriched (data not shown). We next tested whether the $H M L$-E enrichment was dependent on the $\mathrm{D}$ element. We reasoned that if Sum 1 bound the D element in vivo, there should be no antibody-specific enrichment of $H M L$-E in a strain deleted for the D element. To this end, we performed ChIP analysis in a strain with an additional deletion of $H M L$-D. In these experiments the difference in $H M L$-E enrichment in the fractions with or without antibody was indistinguishable (Fig. 3E, $\Delta \mathrm{D}$ ).

The enrichment of Sum 1 at $H M L$-E was consistently weaker than that of Sum1 at the SMK1 promoter. One explanation is that there often are multiple Sum1-binding sites at Sum1-repressed meiotic genes (Pierce et al. 2003), whereas $H M L$-E has only one Sum1-binding site. It is also conceivable that Sum $1 \mathrm{ChIP}$ at $H M L$-E for some reason is sterically hindered as compared to $S M K 1$, although Sum 1 enrichment was no stronger at $H M L-E$ in a $\operatorname{sir} 4 \Delta$ strain as compared to wild type. Quantitation showed that $H M L$-E and SMK1 enrichment were threefold and eightfold, respectively (Fig. 3E, right). Also, adding the $6 x m y c$ tag to the $\mathrm{C}$ terminus rather than the $\mathrm{N}$ terminus did not alter the ability to ChIP Sum1 at $H M L$ (data not shown). However, the fact that we observed consistent enrichment, combined with the in vitro binding of Sum1 to $H M L$-E DNA and the effect of sum1D on $H M L \alpha$ silencing strongly suggests that Sum1 bound in vivo to $H M L$-E via the D element.

\section{sum $1 \Delta$ decreased origin function of HML-E}

The presumed Sum1-binding site at the D2 element lies close to the ORC-binding site of $H M L$-E. Interestingly, other protein-binding sites close to ACS sites of replication origins strongly influence the ability of such sequences to initiate replication (Marahrens and Stillman 1992), raising the question whether Suml affected $H M L$-E origin function. In its chromosomal location, $H M L$-E does not initiate, because it is inactivated by replication forks emanating from centromere-proximal origins (Sharma et al. 2001). However, when removed from this context and placed on a plasmid, $H M L$-E has ARS activity, meaning that it confers autonomous replication to plasmids lacking an origin. Sharma et al. (2001) showed that deletion of a sequence stretch including the D element abrogated the ARS activity of $H M L-\mathrm{E}$, indicating that $\mathrm{D}$ was required for ARS function.

We now asked how Sum 1 affected $H M L$-E origin activity by measuring the stability of a plasmid carrying $H M L-E$ as the sole origin of replication in wild-type and 
Irlbacher et al.

sum $1 \Delta$ strains. Noticeably, the sum $1 \Delta$ strain exhibited a more than twofold higher loss rate of the $H M L$-E plasmid than the wild-type strain (Fig. 4A). This suggested that Sum1 was required for full initiation efficiency of $H M L$-E on a plasmid. Furthermore, sum1s strains grew more slowly than wild-type strains when selecting for the $H M L$-E plasmid (data not shown), also indicating that plasmid transmission, probably through reduced

A

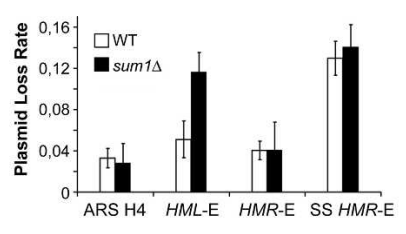

B

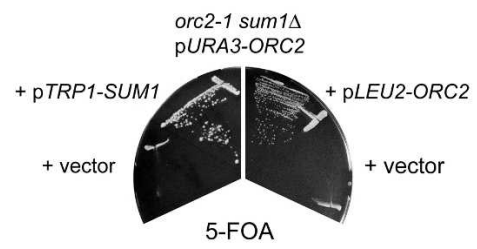

C

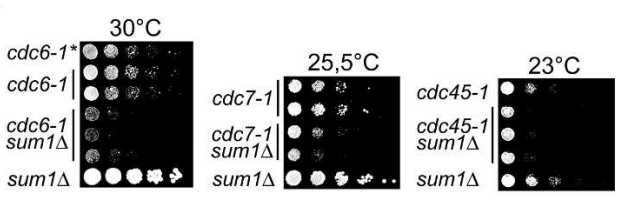

D

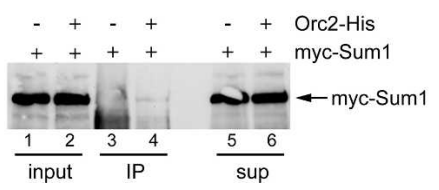

Figure 4. Genetic interactions between SUM1 and replication initiation components. (A) sum $1 \Delta$ reduced the ARS activity of $H M L$-E. Plasmid loss rates were determined in a wild-type (AEY2) and a sum1s (AEY3358) strain. Strains with plasmids carrying ARS H4 (pRS316), HML-E (pAE1119), HMR-E (pAE229), or the HMR-E synthetic silencer SS HMR-E (pAE298) as their sole origins were analyzed. The average loss rates obtained from three independent experiments are shown with corresponding error bars. (B) Synthetic lethality of orc2-1 and

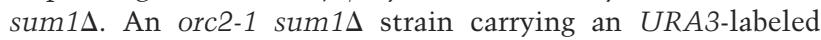
ORC2 plasmid (pRS316-ORC2) was transformed with a SUM1 (pAE1032) or an ORC2 (pAE53) plasmid or the corresponding empty vectors. Its ability to lose the pURA3-ORC2 plasmid was tested on 5-FOA medium. $(C)$ Synthetic growth defects of cdc6-1, cdc7-1, or cdc45-1 with sum1s. Serial dilutions of several segregants from each cross were plated and incubated at the semipermissive temperature of the respective $c d c$ single mutant. For cdc6-1, strains AEY600, 3358, and AEY3537 to 3541, for $c d c 7-1$ strains AEY3542 to 3546, and for $c d c 45-1$ stains AEY373 and AEY3548 to 3551 were used. Incubation was $3 \mathrm{~d}$ for cdc6-1 and cdc7-1 and $6 \mathrm{~d}$ for cdc45-1. cdc6-1 labeled with an asterisk indicates the parental strain, which was not isogenic to the sum1s strain. $(D)$ Coimmunoprecipitation of Sum1 and Orc2. Strains AEY1558 (-) and AEY3474 (6xHis-Orc2, +) carried a $6 x m y c-S u m 12 \mu$ plasmid (pAE1032) and a $H M L \alpha($ pAE1123) $2 \mu$ plasmid. Precipitates were analyzed by SDS-PAGE and immunoblotting using anti-myc-antibody. (Lanes 1,2) Input. (Lanes 3,4) Immunoprecipitation (IP). (Lanes 5,6) Supernatant (sup). origin initiation, was impaired. In contrast, sum1s did not affect the stability of plasmids carrying the wild-type or synthetic $H M R$-E silencers as origins. Also, sum $1 \Delta$ did not affect plasmid stability of an ARS H4 plasmid (Fig. 4A). These results showed that sum1s did not affect other plasmid functions, for instance, CEN function. Also, the effect of sum1s was restricted to $H M L-E$, which was predicted because the D element is not found in the other origins tested. Furthermore, it showed that sum1s did not simply impair weak origins of replication (like the synthetic HMR-E silencer). In summary, these results demonstrated that Sum1 showed a specific effect on origin function of $H M L$-E.

sum $1 \Delta$ interacted genetically with orc mutations, cdc6-1, cdc7-1, and cdc45-1

The plasmid maintenance defect of sum $1 \Delta$ strains with an $H M L$-E-origin plasmid likely reflects a role of Sum 1 in replication initiation at this origin. This observation prompted us to ask whether Suml might be required more globally for replication initiation and thus might constitute a novel replication initiation factor that aids ORC in initiation at selected chromosomal origins. Significantly, we observed that sum1s caused lethality in strains with mutations in the ORC subunits Orc2 and Orc5, since we were unable to recover double mutants in genetic crosses between sum1s and orc2-1 or orc5-1 strains (data not shown), which was in agreement with Suter et al. (2004). The orc mutants on their own are temperature sensitive and show reduced firing of chromosomal origins and high plasmid loss (Fox et al. 1995; Loo et al. 1995). sum1s orc2-1 double mutants were able to grow when provided with a URA3-labeled plasmid carrying $O R C 2$. However, they were only able to survive on URA3-counterselective medium (5-fluoro-orotic acid, 5-FOA) when supplemented with plasmids carrying either SUM1 or ORC2 (Fig. 4B), showing that the lethality depended on these two genes and that sum1s orc2-1 strains were not inviable due to a germination defect. One interpretation of the synthetic interaction between ORC and SUM1 is that chromosomal replication initiation in the orc mutants is further impaired by the absence of Sum 1 such that the cells are unable to survive.

We further assessed genetic interactions between sum1s and mutations in genes encoding other factors required for replication initiation (for review, see Bell and Dutta 2002). Cdc6 is required in early G1 for chromatin binding of MCM proteins and formation of the prereplicative complex (pre-RC) at origins of replication. Cdc7 is part of the DDK (Dbf4 dependent kinase) that is required for the G1/S-phase transition, perhaps by phosphorylating MCM proteins. Cdc45 plays an important role in the transition from initiation to replication. It is required for association of the DNA polymerases with chromatin and colocalizes with the polymerases at the replication fork. We found that double-mutant strains of sum1s with temperature-sensitive alleles of CDC6, $C D C 7$, and CDC45 were viable, but showed a growth defect as compared to the single mutants at their respec- 
tive semipermissive growth temperature (Fig. 4C). Since these mutations impair replication initiation, our findings further supported the notion that Suml played a global role in initiation.

Our observation of a role for Sum 1 in replication initiation and the genetic interaction between sum1s and orc mutations is further bolstered by previous observations of a weak interaction between Orc3 and Sum1 in vivo (Sutton et al. 2001). We further extended this finding in that we observed coimmunoprecipitation between Orc2 and Sum1 (Fig. 4D). This supports the notion that ORC and Suml interact physically at origins of replication.

Sum1 was a replication initiation factor for several origins of replication

A global role for Sum1 in replication initiation predicts that replication origins exist that are also binding sites for Sum1. To search for such sequences, we used the data from two previous studies that identified genome-wide Sum1- and ORC-binding sites using ChIP-mediated microarray analysis (Wyrick et al. 2001; Lee et al. 2002). In both experiments, the authors used the same error model to convert the observed $\mathrm{Cy} 5 / \mathrm{Cy} 3$ intensity ratios into $p$-values (the probability that such a ratio or larger could be observed from a nonbinding event). In their large-scale analysis, they imposed a strict prescription $(p<0.001)$ to reduce the number of wrong binding predictions (false positives) at the expense of a higher false-negative rate (discarding true binding events). For our purposes, we considered those eight intergenic regions where $p($ Sum 1$)<0.01$ and $p($ ORC $)<0.05$ (Fig. 5A). Of these, five were located next to a gene that is derepressed in sum1s as determined by Pierce et al. (2003), suggesting that they constitute true Sum1-binding regions. One (iYJL038C) identified a known ARS, ARS1013 (Wyrick et al. 2001). We asked whether the ARS activity of ARS1013 was affected by Sum 1 by testing ARS function of three overlapping ARS1013 fragments (Wyrick et al. 2001) in wildtype and sum1s strains (Fig. 5B,C). Two fragments (ARS1013-1, ARS1013-2) formed pin-prick transformants that failed to grow upon restreaking (data not shown). In contrast, ARS1013-3, which contains several Sum1-binding sites, formed small transformants in wildtype strains and pin-prick transformants in sum1s strains. Furthermore, the wild-type transformants formed colonies upon restreaking, whereas the sum $1 \Delta$ transformants did not (Fig. 5C). This demonstrated that ARS function of ARS1013 was improved by the presence of Sum1-binding sites and depended on SUM1. Another ARS adjacent to ARS1013, ARS1012, is an active origin of replication (Raghuraman et al. 2001), but does not contain Sum1-binding sites closeby (Fig. 5B). When tested for plasmid maintenance, ARS1012 transformants grew equally well in wild-type and sum1s strains (Fig. 5C). Taken together, these experiments showed that Sum1binding sites within a replicator improved origin function.

To further test this notion, we next determined
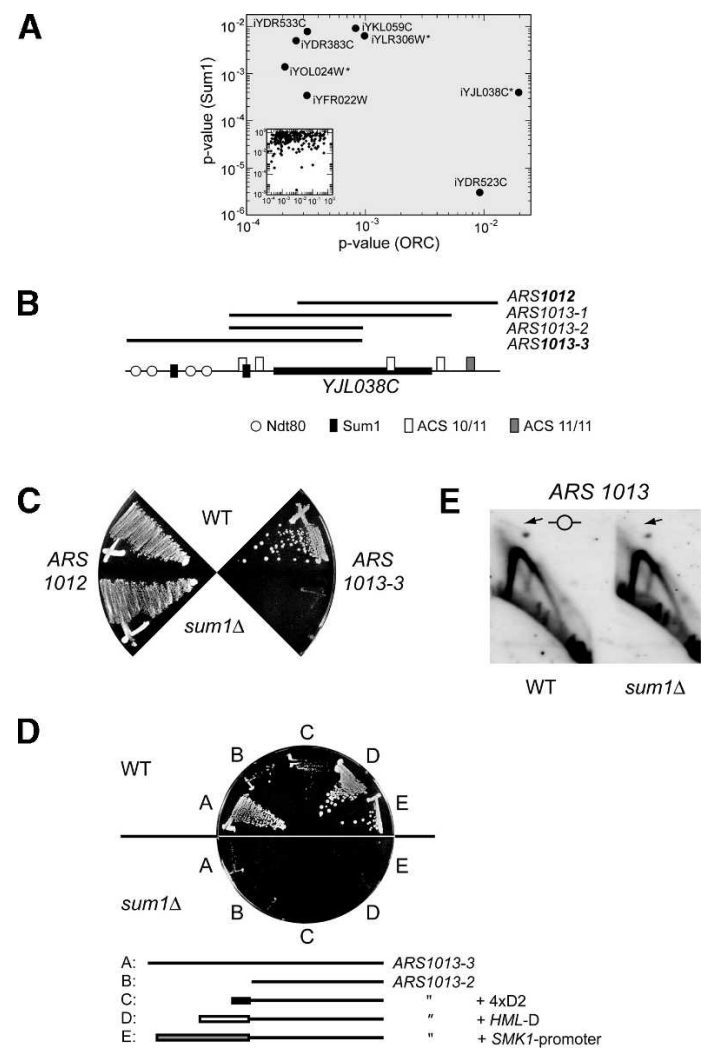

Figure 5. Sum 1 was a replication initiation factor. $(A)$ Plot of $p$-values for Sum 1 binding (Lee et al. 2002), $p<0.01$ versus ORC binding (Wyrick et al. 2001), $p<0.05$. (Inset) All data points. The origin function of intergenic regions labeled with an asterisk was tested below. (B) Schematic representation of ARS1012 and ARS1013 located at the ORF YJLO38C on chromosome X. The location of Ndt80 and Sum 1 consensus sites (Pierce et al. 2003) and ACS matches is indicated. Bold lines represent fragments whose ARS function was tested. (C) SUM1 was required for ARS activity of ARS 1013 on plasmids. Strains AEY2 (WT) and AEY3358 (sum1A) were transformed with URA-CEN4 plasmids carrying either ARS1012 (pAE1076) or ARS1013-3 (pAE1081) as their sole origin. Transformants obtained upon transformation of ARS1013-1 or ARS1013-2 -URA-CEN4 plasmids (pAE1078, pAE1080) were not restreakable. (D) SUM1 was required for chromosomal origin activity of ARS1013. The appearance of bubble-shaped replication intermediates indicative of chromosomal initiation (arrows) was measured by 2D gel electrophoresis and Southern hybridization in a wild-type (AEY2) and sum1s (AEY3358) strain. (E) Addition of Sum1binding sites improved the ARS function of ARS1013-2. Strains AEY2 (WT) and AEY3358 (sum1A) were transformed with URA-CEN4 plasmids either carrying ARS1013-3 (pAE1081) or variants of ARS1013-2 containing additional fragments of $H M L$-E $\left(4 \mathrm{xD} 2, \mathrm{pAE} 1159\right.$ and $\left.H M L-\mathrm{E} \mathrm{ACS}^{-}, \mathrm{pAE} 1160\right)$ or the SMK1 promoter (pAE1161) upstream of the ARS1013-2 fragment.

whether Sum 1 sites other than those naturally present at ARS1013 could improve ARS function of a weak origin. This was achieved by adding ectopic Sum1-binding sites from $H M L-\mathrm{D}$ (4xD2 or $H M L-\mathrm{D})$ or the $S M K 1$ promoter to ARS1013-2 and testing ARS function in wild-type and sum1s strains. Addition of $H M L-D$ or the $S M K 1$ pro- 
moter significantly improved ARS function, and the improvement was completely dependent on Sum1 (Fig. 5D), which showed that Sum 1 sites form alternative sources had the ability to increase initiation of a plasmid origin. Addition of four D2 elements barely increased initiation, suggesting that the D2 element was too minimal for Sum1 binding in this context.

Our observation that Sum 1 affected plasmid stability suggested that it might also affect chromosomal replication initiation of Sum1-binding origins. To investigate this, we measured origin firing of ARS1013 in its native chromosomal location in wild-type and sum1s strains by performing two-dimensional origin mapping gels (Fangman and Brewer 1991). We observed a weak signal indicative of bubble-shaped replication intermediates in the wild-type strain (Fig. 5E, arrow), indicating that ARS1013 was an inefficient chromosomal origin. This was expected, because ARS1013 lies close to ARS1012, which has stronger ARS activity than ARS1013 and therefore probably initiates in the majority of cell cycles and inactivates ARS1013. However, this signal was absent in the sum1s strain (Fig. 5E). This showed that Sum 1 was required for replication initiation of ARS1013 in its chromosomal environment.

We also determined the plasmid maintenance properties of two other intergenic regions from our data set, iYLR306W (ARS1223) and iYOL024W (ARS1511). Both were designated "proposed ARS" (pro-ARS) by Wyrick et al. (2001) due to their ability to bind ORC and $\mathrm{Mcm}$ proteins. However, their ARS activity so far has not been tested. We selected these regions, because they colocalize with probable in vivo origins of replication as determined by genome-wide density transfer experiments (Raghuraman et al. 2001). In a plasmid maintenance assay, we found that ARS1223 and ARS1511 indeed conferred autonomous replication to an origin-less plasmid, and that they displayed a significantly increased plasmid loss rate in sum1s cells as compared to wild-type cells (Fig. 6A). This showed that the replication capacity of these origins depended on Sum1.

In a complementary approach, we used a binding-motif-based sequence search to find origins that require Sum1 for full activity. Using the consensus sequence for Sum 1 binding (DSYGWCAYWDW), we searched the genome for regions where Sum1-binding sites and ACS sites lie within a distance of $<200 \mathrm{bp}$. Among these was the known ARS606. Significantly, we observed that ARS activity of ARS606 strongly depended on Sum1, since sum1s transformants containing this ARS did not grow upon restreaking, whereas wild-type transformants did (Fig. 6B). In summary, these results showed that multiple origins within the yeast genome required Sum 1 for full initiation potential, indicating that Sum1 was a general initiation factor.

\section{Discussion}

Heterochromatin targeting and replication initiation are mechanistically linked by the observation that ORC, the replication initiator, is required for both processes. Here,
A

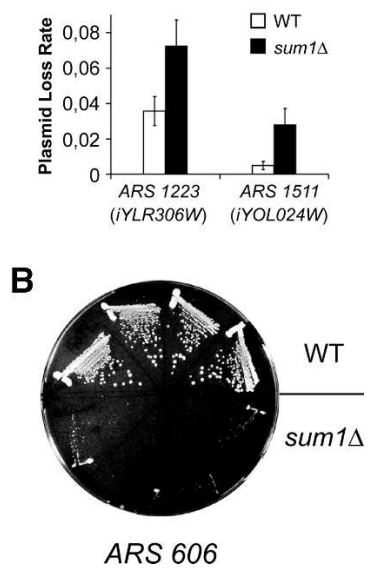

Figure 6. Sum 1 affected ARS activity of selected origins of replication. (A) ARS1223 and ARS1511 required SUM1 for full ARS activity. Plasmid loss rates were determined in a wild-type (WT; AEY2) and a sum1D (AEY3358) strain. Strains with URACEN4 plasmids carrying ARS1223 (pAE1130) or ARS1511 (pAE1135) as their sole origins were analyzed. The average loss rates obtained from three independent experiments are shown with corresponding error bars. The loss rate in sum1s strains was approximately twofold (ARS1223) and 5.7-fold (ARS1511) higher than in wild-type strains. (B) ARS activity of ARS606 was dependent on SUM1. Strains AEY2 (WT) and AEY3358 (sum1D) were transformed with URA-CEN4 plasmids carrying ARS606 (pAE1126) as their sole origin and streaked on a -Ura plate.

we found that the Sum1 protein bound to a functional element of the $H M L$-E silencer and was required for $H M L$ silencing as well as for replication initiation at $H M L$-E and several origins of replication in the yeast genome. Thus, Sum1 represents a novel regulator of replication in yeast. In this function, Sum 1 may be comparable to Abf1 (Diffley and Stillman 1988; Eisenberg et al. 1988), Rap1 (Kimmerly et al. 1988), or Mcm1 (Chang et al. 2004), which bind to a subset of yeast origins and are required for efficient initiation. A picture emerges where yeast replication origins, in addition to ORC, bind an accessory factor that enhances initiation, with different subsets of origins being bound by different modulators.

How does Sum1 promote replication initiation? We show that Sum 1 binds to origins in the vicinity of ORC and interacts physically with ORC. Sum 1 binding close to origins may affect nucleosome positioning at the ACS, thus providing a more favorable environment for ORC binding and increasing the likelihood of initiation (Lipford and Bell 2001). Sum 1 may act before pre-RC formation and thus may be necessary for early initiation events. Alternatively, it might function downstream of the pre-RC, for instance, in the transition from initiation to replication. Such a role of Sum1 might be similar to Drosophila Myb (see below), which affects replication initiation, but not the formation of pre-RCs (Beall et al. 2002). Furthermore, Sum 1 may recruit the HDAC Hst1 to origins, such that its influence on initiation may be exerted through chromatin deacetylation, a notion that is supported by the synthetic sick phenotype of an orc2-1 
hst1s strain (Suter et al. 2004). Thus, Hst1 in cooperation with Sum1 may influence both gene repression and origin firing by locally deacetylating histones. Interestingly, two other HDACs have been implicated in initiation: The absence of Rpd3 deacetylation causes late origins to fire early (Vogelauer et al. 2002; Aparicio et al. 2004), and Sir2 has a negative role in initiation at selected origins (Pappas et al. 2004). Thus, the different HDACs seem to have highly individual effects on their target origins.

The observation of synthetic phenotypes between orc or $c d c$ mutations and sum $1 \Delta$ suggests that sum1s, although on its own not lethal (Chi and Shore 1996), compromises replication initiation such that it is incompatible with reduced initiation. That the phenotypes are more severe with orc than with the $c d c$ mutations may point toward a direct function of Sum 1 with ORC, but could also be due to differences in severity of the mutant alleles. It is also possible that sum $1 \Delta$ additionally affects other processes that become essential in orc mutants, for instance, sister chromatid cohesion (Suter et al. 2004). A global effect for sum1s on initiation suggests that the number of Sum1-modulated origins must be sufficiently high to cause cell death in orc2-1 sum1s mutants, but our predicted set of possible Sum1-affected origins shows only few such origins. However, our mode of prediction was quite stringent: In addition to a requirement to be bound by both ORC and Sum1, we only scored origins upstream of genes that were derepressed in a sum1s strain in our first approach (Pierce et al. 2003). Thus, several parameters restricted our origin identification: (1) The ChIP-on-chip analysis for ORC-binding sites has probably not identified all sites, since Breier et al. (2004) found sequences by computational analysis that were not in the ORC-binding data set (Wyrick et al. 2001) but were active origins in the ARS assay. This is also reflected by the fact that we found another Sum1-dependent ARS, ARS606, by an independent search for Sum1and ORC-binding site colocalization. (2) Equally, the $p$-value prescription of the binding experiment may also exclude intergenic regions with real binding of Sum 1 . For instance, one known Sum1-binding site, the MSE within the SMK1 promoter (Xie et al. 1999), is not identified by this data set (Lee et al. 2002). Also, microarray analysis may only be sensitive enough to find locations with multiple Sum1-binding sites, as is the case for many Sum1-regulated genes (Pierce et al. 2003), whereas origins may contain only one Suml-binding site, as is the case for $H M L$-E. (3) There may be Sum1-binding sites that do not regulate the neighboring gene, but may be part of an origin. (4) The Sum1-binding site may be at a longer distance from the ACS. (5) Origins with co-occurrence of ORC and Sum1 binding may also lie within coding regions. Taken together, it seems likely that several more Sum1-regulated origins exist that await identification.

So far, Sum 1 was solely considered a repressor of meiotic genes. Our work now demonstrates that Sum 1 has a global function in replication initiation. One notable aspect about the involvement of Sum 1 in replication is its regulation during meiosis. While constant throughout the mitotic cell cycle, Sum 1 protein levels dramatically decrease during the early stages of meiosis, concurrently with premeiotic $S$ phase, and are lowest in the middle stages (Lindgren et al. 2000). This raises the question of how Suml-affected origins initiate in premeiotic replication. Perhaps the absence of Sum1 leads to a delayed or a reduced firing rate at selected origins, and origin usage thus may be reduced in meiotic cells, which is in agreement with the observation that sum $1 \Delta$ diploids progress slightly slower than wild type into meiosis (Lindgren et al. 2000). Whether Suml affects premeiotic replication will depend upon how mechanistically it functions in initiation. For instance, if it is required for pre-RC formation, Suml levels at the time of pre-RC formation must be low enough to inhibit the process.

In this study, we furthermore identified wild-type Suml as an anchoring factor for heterochromatin at $H M L$. This finding extends previous work that had solely recognized a function for the mutant Sum1-1, but not for wild-type Sum1, in silencing. Interestingly, whereas Sum1-1 recruits Hst1 instead of Sir2 to establish repression (Rusche and Rine 2001; Sutton et al. 2001), Sum 1 at $H M L$ apparently does not. Perhaps Sum1 binds differently to $H M L$-D than it does to MSE elements, such that it now preferentially recruits Sir2 rather than Hst1 to the $H M L$ locus. That Sum1 can function without Hst1 is reflected in the finding that Sum 1 can also exert its repressive function independently of Hst 1 at a subset of middle-meiotic genes (McCord et al. 2003). In its silencing role, Sum 1 most likely interacts with other silencing factors, for instance, the Sir silencing complex, to stabilize the nucleation of silent chromatin at HML. Sum 1 so far has been known as a local, gene-specific repressor of meiotic genes (Xie et al. 1999). The fact that Sum1 is repressed in meiosis, which in yeast is induced by depriving cells of glucose, and that Sum 1 is required for $H M L$ silencing, jibes with an earlier, elegant observation that silencing can be made dependent on the environmental conditions (Shei and Broach 1995). In this study, $H M$ silencers transposed to the MAT locus could elicit silencing on glucose-containing medium, but this silencing was relieved on nonfermentable carbon sources such as are used to induce meiosis. In light of our results, one interpretation of this observation is that Sum 1 is no longer present under these conditions, such that silencing is abrogated. Interestingly, other silencer-binding proteins like Rap1 and Abf1 function as transcriptional activators rather than as repressors elsewhere in the genome (Shore and Nasmyth 1987; Halfter et al. 1989). This situation is paralleled in higher eukaryotes in that the recruitment of Polycomb group complexes to Polycomb response elements (PREs) to maintain homeotic gene repression involves proteins like GAGA and Pho that can function as transcriptional activators as well as repressors (Kerrigan et al. 1991; Brown et al. 1998).

On a broader perspective, the finding that a factor whose expression is regulated by the cell program (i.e., meiosis vs. mitosis) influences replication initiation and silencing in yeast, can be compared to the way multicel- 
lular organisms exercise control over replication and heterochromatin formation during development. Metazoans use differential origin patterns to replicate a given chromosomal area depending on the cell type. For example, Drosophila embryonic cells have a much broader use of origins than cells of later stages, probably in order to complete the early cell cycles faster than in more differentiated cells, which must accommodate their cell cycle to the respective tissue environment (Sasaki et al. 1999). Also, the spacing between meiotic origins in the newt Triturus cristatus is much longer than in mitotic cells, and accordingly, premeiotic S phase is substantially longer than the mitotic S phase (Callan 1974). The function of Sum 1 at yeast origins may be analogous to that of Drosophila Myb at replication origins in the chorion loci of follicle cells, where Myb is required for sitespecific DNA replication leading to gene amplification (Beall et al. 2002). Perhaps other eukaryotic replication modulators exist that are functionally related to Sum1, are expressed in the early stages of development, and which, in cooperation with ORC, activate origins that are silent in their absence. The down-regulation of these hypothesized factors would reduce origin usage, thus contributing to the lengthening of the cell cycle by increasing the distance between origins. Conversely, origins could be activated differentially in specialized cell types or in meiosis by regulating the expression of origin accessory factors. In summary, the modulation of heterochromatinization and replication initiation by regulating an accessory factor could constitute an economical way for an organism to control origin usage and heterochromatin formation during development and differentiation.

In conclusion, we propose a model for the regulation of origin choice and usage as well as heterochromatin formation during meiosis and differentiation. We present data that a factor that is repressed in meiosis is required for replication initiation at several origins and for gene silencing in yeast. We propose that larger eukaryotes use this mechanism of regulating an accessory factor to differentially control replication and the chromatin state of their genome during different stages of development. A future challenge will be to identify such eukaryotic regulators and to investigate how they integrate the processes of replication initiation and heterochromatin formation.

\section{Materials and methods}

Yeast strains and plasmids are described in the Supplemental Material.

\section{Silencing assays}

Qualitative and quantitative mating assays were performed as described (Ehrenhofer-Murray et al. 1997) using AEY264 (MATa his4) and AEY265 (MAT $\alpha$ his4) as mating tester strains. All quantitative mating efficiencies are the average of at least two independent determinations and were normalized to the wildtype strain AEY 2.

Plasmid loss rates

Plasmid loss rates were determined in strains AEY2 and AEY3358 (sum1s:: HisMX) according to Dillin and Rine (1997).
The loss rate is equivalent to the fraction of daughter cells that have received no plasmids during the previous cell division.

\section{EMSAs}

6xHis-Sum1 used for EMSAs was prepared from BL21 Codon Plus cells transformed with pAE1054. Cells were grown to mid$\log$ phase, protein expression was induced by addition of isopropyl- $\beta$-D-thiogalactopyranoside (IPTG) to $0.5 \mathrm{mM}$, and cells were allowed to grow overnight at $15^{\circ} \mathrm{C}$. Cell lysis was carried out by sonication, and the protein was purified using a Ni-NTA column (QIAGEN). The protein was eluted with $250 \mathrm{mM}$ imidazole and the eluate was dialyzed overnight at $4^{\circ} \mathrm{C}$ against $20 \mathrm{mM}$ Tris- $\mathrm{HCl}(\mathrm{pH} 8), 50 \mathrm{mM} \mathrm{NaCl}$, and $1 \mathrm{mM}$ EDTA. To increase the protein concentration, centricon tubes (Millipore) were used according to the manufacturer's guidelines. Probe preparation and EMSAs were carried out as previously described (Xie et al. 1999). For EMSAs, PCR fragments of the respective regions were amplified from AEY2 (for the HML-E wild type and the INO1 sequence), AEY3395 (for the HML-E ACS ${ }^{-}$sequence), AEY3391 (for the $H M L$-E D $\Delta$ sequence), AEY3398 (for the $H M L$-E ACS $\mathrm{D} \Delta$ sequence), or AEY3404 (for the HML-E D2- sequence) and were purified and labeled as described (Xie et al. 1999). The primer sequence for the $H M L$-E wild-type and $H M L-E \mathrm{ACS}^{-}$ PCR reaction was GGTGTATCGCAATGGAATG (HML-E up) and CCCGAAATCGATAATAA (HML-E down). The reverse primer for the $H M L-\mathrm{E} \mathrm{ACS}^{-} \mathrm{D} \Delta$ PCR reaction was GTTTA CATTTCATTCTATGTGCGCTAG (HML-E downII). For the INO1 PCR product, primers TGTTCTGTTGTCGGGTTCC (INOup) and GTAGTCTTGAACAGTGGGCG (INOdown) were used. For Figure 3C, PCR primers for $H M L$-E wild-type and $H M L$-E D2 $\Delta$ were GGGTTTTTGATTTTTTTATGTTTTTTT TAAAACATTAAAG (HML-EACSfw) and HML-Edown. For $H M L$-E D $\Delta$, primers were GGGTTTTTGATTTTTTTATGTTT TTTTTAAATCGATTTCG (HML-E $\mathrm{D}^{-} \mathrm{fw}$ ), and HML-E downII. For SMK1 sequence binding, the oligonucleotide sequence was CCACTAATTTGTGACACTT (with corresponding antiparallel oligonucleotide).

\section{ChIPs}

ChIPs were performed essentially as described (Rusche and Rine 2001), except that mouse anti-myc antibody (Invitrogen) at $4 \mu \mathrm{g}$ per sample and protein-G Sepharose beads were used. Crosslinking was carried out in $1 \times$ TBS with $10 \mathrm{mM}$ dimethyl-adipimidate (DMA, Pierce) for $45 \mathrm{~min}$ at room temperature and subsequently in $1 \times$ TBS with $1 \%$ formaldehyde for 30 min (Kurdistani and Grunstein 2003). PCR reactions were performed using 1.25 units of Taq DNA polymerase (Promega), with $3 \mathrm{mM}$ $\mathrm{MgCl}_{2}, 0.25 \mathrm{mM}$ dNTPs, and $0.5 \mu \mathrm{M}$ each primer. Samples were cycled 28 times for $15 \mathrm{sec}$ at $94^{\circ} \mathrm{C}, 20 \mathrm{sec}$ at $54^{\circ} \mathrm{C}$, and $2.5 \mathrm{~min}$ at $72^{\circ} \mathrm{C}$. The oligonucleotides used are described in Rusche and Rine (2001), except $H M L$-EdownII (GTTTACATTTCATTC TATGTGCGCTAG), which was used as a reverse primer for $H M L$-E sequence amplification.

\section{Immunoprecipitations}

Yeast extracts from the protease-deficient strains AEY1558 and AEY3474 were prepared as follows: Per coimmunoprecipitation experiment, $50 \mathrm{OD}$ of cells were suspended in $500 \mu \mathrm{L}$ of lysis buffer $(50 \mathrm{mM}$ Tris- $\mathrm{HCl}$ at $\mathrm{pH} 7.5,140 \mathrm{mM} \mathrm{NaCl}, 1 \%$ Triton X-100, 0.1\% ND-40, 1 mM PMSF, 1× "complete" proteinase inhibitor [Roche Diagnostics], $1 \mathrm{mM}$ DTT) and disrupted with glass beads for $5 \mathrm{~min}$ using a vortex mixer. The lysate was separated by centrifugation. Antibody ( $\alpha$-myc [Invitrogen] or 
$\alpha$-poly-His [Sigma]) was added to the lysate and incubated overnight at $4^{\circ} \mathrm{C}$ with shaking. Subsequently, G-Sepharose 4-FF beads (Pharmacia) were added to the lysate-antibody mix and incubated for $1 \mathrm{~h}$. Immunoprecipitates were collected by brief centrifugation and washed three times with lysis buffer. The resulting precipitate was resuspended in SDS sample buffer and analyzed by SDS-PAGE and immunoblotting according to standard protocols.

\section{Two-dimensional origin mapping gels}

Replication intermediates were isolated and analyzed as described (Fangman and Brewer 1991). DNA was digested with BfuAI, which generates a $4.25-\mathrm{kB}$ fragment of ARS1013. ARS1013 was detected using two probes: a 1.2-kB EcoRI-HindIII fragment of pAE1078 and a 380-bp EcoRI-SacI fragment of pAE1081.

\section{Acknowledgments}

We are grateful to O. Aparicio, J. Broach, J. Huberman, B. Jones, D. Koshland, J. Rine, D. Shore, and R. Sternglanz for generously providing strains and plasmids. We thank R. Kamakaka and W. Messer for critical reading of the manuscript, U. Marchfelder and M. Müller for technical assistance, and our laboratory for many stimulating discussions. This research was supported by a grant to A.E.E.-M. from the Deutsche Forschungsgemeinschaft (EH 194/1-1 to 1-4) and the Max-Planck Society. T.M. acknowledges funding by European Community Contract No. QLRI-CT-2001-00015.

\section{References}

Aparicio, J.G., Viggiani, C.J., Gibson, D.G., and Aparicio, O.M. 2004. The Rpd3-Sin3 histone deacetylase regulates replication timing and enables intra-S origin control in Saccharomyces cerevisiae. Mol. Cell. Biol. 24: 4769-4780.

Beall, E.L., Manak, J.R., Zhou, S., Bell, M., Lipsick, J.S., and Botchan, M.R. 2002. Role for a Drosophila Myb-containing protein complex in site-specific DNA replication. Nature 420: $833-837$.

Bell, S.P. and Dutta, A. 2002. DNA replication in eukaryotic cells. Annu. Rev. Biochem. 71: 333-374.

Bell, S.P., Kobayashi, R., and Stillman, B. 1993. Yeast origin recognition complex functions in transcription silencing and DNA replication. Science 262: 1844-1849.

Breier, A.M., Chatterji, S., and Cozzarelli, N.R. 2004. Prediction of Saccharomyces cerevisiae replication origins. Genome Biol. 5: R22.

Brown, J.L., Mucci, D., Whiteley, M., Dirksen, M.L., and Kassis, J.A. 1998. The Drosophila Polycomb group gene pleiohomeotic encodes a DNA binding protein with homology to the transcription factor YY1. Mol. Cell 1: 1057-1064.

Callan, H.G. 1974. DNA replication in the chromosomes of eukaryotes. Cold Spring Harb. Symp. Quant. Biol. 38: 195203.

Chang, V.K., Donato, J.J., Chan, C.S., and Tye, B.K. 2004. Mcm1 promotes replication initiation by binding specific elements at replication origins. Mol. Cell. Biol. 24: 6514-6524.

Chi, M.H. and Shore, D. 1996. SUM1-1, a dominant suppressor of SIR mutations in Saccharomyces cerevisiae, increases transcriptional silencing at telomeres and HM mating-type loci and decreases chromosome stability. Mol. Cell. Biol. 16: 4281-4294.

Diffley, J.F. and Stillman, B. 1988. Purification of a yeast protein that binds to origins of DNA replication and a transcriptional silencer. Proc. Natl. Acad. Sci. 85: 2120-2124.

Dillin, A. and Rine, J. 1997. Separable functions of ORC5 in replication initiation and silencing in Saccharomyces cerevisiae. Genetics 147: 1053-1062.

Ehrenhofer-Murray, A.E., Gossen, M., Pak, D.T., Botchan, M.R., and Rine, J. 1995. Separation of origin recognition complex functions by cross-species complementation. Science 270: 1671-1674.

Ehrenhofer-Murray, A.E., Rivier, D.H., and Rine, J. 1997. The role of Sas2, an acetyltransferase homologue of Saccharomyces cerevisiae, in silencing and ORC function. Genetics 145: 923-934.

Eisenberg, S., Civalier, C., and Tye, B.K. 1988. Specific interaction between a Saccharomyces cerevisiae protein and a DNA element associated with certain autonomously replicating sequences. Proc. Natl. Acad. Sci. 85: 743-746.

Fangman, W.L. and Brewer, B.J. 1991. Activation of replication origins within yeast chromosomes. Annu. Rev. Cell Biol. 7: 375-402.

Feldman, J.B., Hicks, J.B., and Broach, J.R. 1984. Identification of the sites required for repression of a silent mating type locus in yeast. J. Mol. Biol. 178: 815-834.

Foss, M., McNally, F.J., Laurenson, P., and Rine, J. 1993. Origin recognition complex (ORC) in transcriptional silencing and DNA replication in S. cerevisiae. Science 262: 1838-1844.

Fox, C.A., Loo, S., Dillin, A., and Rine, J. 1995. The origin recognition complex has essential functions in transcriptional silencing and chromosomal replication. Genes \& Dev. 9: 911-924.

Geissenhoner, A., Weise, C., and Ehrenhofer-Murray, A.E. 2004. Dependence of ORC silencing function on NatA-mediated $\mathrm{N} \alpha$ acetylation in Saccharomyces cerevisiae. Mol. Cell. Biol. 24: 10300-10312.

Halfter, H., Kavety, B., Vandekerckhove, J., Kiefer, F., and Gallwitz, D. 1989. Sequence, expression and mutational analysis of BAF1, a transcriptional activator and ARS1-binding protein of the yeast Saccharomyces cerevisiae. EMBO I. 8: 4265-4272.

Hecht, A., Laroche, T., Strahl-Bolsinger, S., Gasser, S.M., and Grunstein, M. 1995. Histone H3 and H4 N-termini interact with SIR3 and SIR4 proteins: A molecular model for the formation of heterochromatin in yeast. Cell 80: 583-592.

Hoppe, G.J., Tanny, J.C., Rudner, A.D., Gerber, S.A., Danaie, S., Gygi, S.P., and Moazed, D. 2002. Steps in assembly of silent chromatin in yeast: Sir3-independent binding of a Sir2/Sir4 complex to silencers and role for Sir2-dependent deacetylation. Mol. Cell. Biol. 22: 4167-4180.

Imai, S., Armstrong, C.M., Kaeberlein, M., and Guarente, L. 2000. Transcriptional silencing and longevity protein Sir2 is an NAD-dependent histone deacetylase. Nature 403: 795 800.

Kerrigan, L.A., Croston, G.E., Lira, L.M., and Kadonaga, J.T. 1991. Sequence-specific transcriptional antirepression of the Drosophila Kruppel gene by the GAGA factor. J. Biol. Chem. 266: 574-582.

Kimmerly, W., Buchman, A., Kornberg, R., and Rine, J. 1988. Roles of two DNA-binding factors in replication, segregation and transcriptional repression mediated by a yeast silencer. EMBO I. 7: 2241-2253.

Kurdistani, S.K. and Grunstein, M. 2003. In vivo protein-protein and protein-DNA crosslinking for genomewide binding microarray. Methods 31: 90-95.

Laurenson, P. and Rine, J. 1991. SUM1-1: A suppressor of silencing defects in Saccharomyces cerevisiae. Genetics 129: 685-696. 
Lee, D.G. and Bell, S.P. 1997. Architecture of the yeast origin recognition complex bound to origins of DNA replication. Mol. Cell. Biol. 17: 7159-7168.

Lee, T.I., Rinaldi, N.J., Robert, F., Odom, D.T., Bar-Joseph, Z., Gerber, G.K., Hannett, N.M., Harbison, C.T., Thompson, C.M., Simon, I., et al. 2002. Transcriptional regulatory networks in Saccharomyces cerevisiae. Science 298: 799-804.

Lindgren, A., Bungard, D., Pierce, M., Xie, J., Vershon, A., and Winter, E. 2000. The pachytene checkpoint in Saccharomyces cerevisiae requires the Sum 1 transcriptional repressor. EMBO I. 19: 6489-6497.

Lipford, J.R. and Bell, S.P. 2001. Nucleosomes positioned by ORC facilitate the initiation of DNA replication. Mol. Cell 7:21-30.

Livi, G.P., Hicks, J.B., and Klar, A.J. 1990. The sum1-1 mutation affects silent mating-type gene transcription in Saccharomyces cerevisiae. Mol. Cell. Biol. 10: 409-412.

Loo, S., Fox, C.A., Rine, J., Kobayashi, R., Stillman, B., and Bell, S. 1995. The origin recognition complex in silencing, cell cycle progression, and DNA replication. Mol. Biol. Cell. 6: $741-756$.

Mahoney, D.J., Marquardt, R., Shei, G.J., Rose, A.B., and Broach, J.R. 1991. Mutations in the HML E silencer of Saccharomyces cerevisiae yield metastable inheritance of transcriptional repression. Genes \& Dev. 5: 605-615.

Marahrens, Y. and Stillman, B. 1992. A yeast chromosomal origin of DNA replication defined by multiple functional elements. Science 255: 817-823.

McCord, R., Pierce, M., Xie, J., Wonkatal, S., Mickel, C., and Vershon, A.K. 2003. Rfm1, a novel tethering factor required to recruit the Hstl histone deacetylase for repression of middle sporulation genes. Mol. Cell. Biol. 23: 2009-2016.

Pak, D.T., Pflumm, M., Chesnokov, I., Huang, D.W., Kellum, R., Marr, J., Romanowski, P., and Botchan, M.R. 1997. Association of the origin recognition complex with heterochromatin and HP1 in higher eukaryotes. Cell 91: 311-323.

Pappas Jr., D.L., Frisch, R., and Weinreich, M. 2004. The $\mathrm{NAD}(+)$-dependent Sir2p histone deacetylase is a negative regulator of chromosomal DNA replication. Genes \& Dev. 18: 769-781.

Pierce, M., Benjamin, K.R., Montano, S.P., Georgiadis, M.M., Winter, E., and Vershon, A.K. 2003. Sum1 and Ndt80 proteins compete for binding to middle sporulation element sequences that control meiotic gene expression. Mol. Cell. Biol. 23: 4814-4825.

Raghuraman, M.K., Winzeler, E.A., Collingwood, D., Hunt, S., Wodicka, L., Conway, A., Lockhart, D.J., Davis, R.W., Brewer, B.J., and Fangman, W.L. 2001. Replication dynamics of the yeast genome. Science 294: 115-121.

Rhode, P.R., Elsasser, S., and Campbell, J.L. 1992. Role of multifunctional autonomously replicating sequence binding factor 1 in the initiation of DNA replication and transcriptional control in Saccharomyces cerevisiae. Mol. Cell. Biol. 12: 1064-1077.

Rivier, D.H. and Rine, J. 1992. Silencing: The establishment and inheritance of stable, repressed transcription states. Curr. Opin. Genet. Dev. 2: 286-292.

Rivier, D.H., Ekena, J.L., and Rine, J. 1999. HMR-I is an origin of replication and a silencer in Saccharomyces cerevisiae. Genetics 151: 521-529.

Rusche, L.N. and Rine, J. 2001. Conversion of a gene-specific repressor to a regional silencer. Genes \& Dev. 15: 955-967.

Rusche, L.N., Kirchmaier, A.L., and Rine, J. 2002. Ordered nucleation and spreading of silenced chromatin in Saccharomyces cerevisiae. Mol. Biol. Cell 13: 2207-2222.

2003. The establishment, inheritance, and function of silenced chromatin in Saccharomyces cerevisiae. Annu. Rev. Biochem. 72: 481-516.

Sasaki, T., Sawado, T., Yamaguchi, M., and Shinomiya, T. 1999. Specification of regions of DNA replication initiation during embryogenesis in the 65-kilobase DNApol $\alpha$-dE2F locus of Drosophila melanogaster. Mol. Cell. Biol. 19: 547-555.

Sharma, K., Weinberger, M., and Huberman, J.A. 2001. Roles for internal and flanking sequences in regulating the activity of mating-type-silencer-associated replication origins in Saccharomyces cerevisiae. Genetics 159: 35-45.

Shei, G.J. and Broach, J.R. 1995. Yeast silencers can act as orientation-dependent gene inactivation centers that respond to environmental signals. Mol. Cell. Biol. 15: 3496-3506.

Shore, D. and Nasmyth, K. 1987. Purification and cloning of a DNA binding protein from yeast that binds to both silencer and activator elements. Cell 51: 721-732.

Suter, B., Tong, A., Chang, M., Yu, L., Brown, G.W., Boone, C., and Rine, J. 2004. The origin recognition complex links replication, sister chromatid cohesion and transcriptional silencing in Saccharomyces cerevisiae. Genetics 167: 579591.

Sutton, A., Heller, R.C., Landry, J., Choy, J.S., Sirko, A., and Sternglanz, R. 2001. A novel form of transcriptional silencing by Sum 1-1 requires Hst 1 and the origin recognition complex. Mol. Cell. Biol. 21: 3514-3522.

Triolo, T. and Sternglanz, R. 1996. Role of interactions between the origin recognition complex and SIR1 in transcriptional silencing. Nature 381: 251-253.

Vogelauer, M., Rubbi, L., Lucas, I., Brewer, B.J., and Grunstein, M. 2002. Histone acetylation regulates the time of replication origin firing. Mol. Cell 10: 1223-1233.

Wilmes, G.M. and Bell, S.P. 2002. The B2 element of the Saccharomyces cerevisiae ARS1 origin of replication requires specific sequences to facilitate pre-RC formation. Proc. Nat1. Acad. Sci. 99: 101-106.

Wyrick, J.J., Aparicio, J.G., Chen, T., Barnett, J.D., Jennings, E.G., Young, R.A., Bell, S.P., and Aparicio, O.M. 2001. Genome-wide distribution of ORC and MCM proteins in $S$. cerevisiae: High-resolution mapping of replication origins. Science 294: 2357-2360.

Xie, J., Pierce, M., Gailus-Durner, V., Wagner, M., Winter, E., and Vershon, A.K. 1999. Suml and Hst1 repress middle sporulation-specific gene expression during mitosis in Saccharomyces cerevisiae. EMBO I. 18: 6448-6454.

Zou, L. and Stillman, B. 2000. Assembly of a complex containing Cdc45p, replication protein $\mathrm{A}$, and $\mathrm{Mcm} 2 \mathrm{p}$ at replication origins controlled by S-phase cyclin-dependent kinases and Cdc7p-Dbf4p kinase. Mol. Cell. Biol. 20: 3086-3096. 


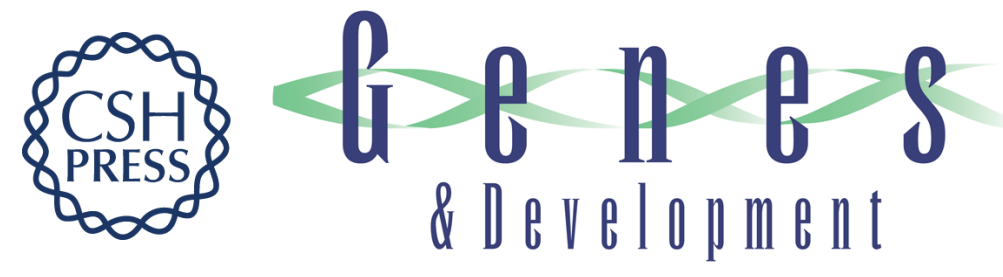

\section{Control of replication initiation and heterochromatin formation in Saccharomyces cerevisiae by a regulator of meiotic gene expression}

Horst Irlbacher, Jacqueline Franke, Thomas Manke, et al.

Genes Dev. 2005, 19:

Access the most recent version at doi:10.1101/gad.334805

Supplemental http://genesdev.cshlp.org/content/suppl/2005/07/29/19.15.1811.DC1
Material

References This article cites 58 articles, 40 of which can be accessed free at:

http://genesdev.cshlp.org/content/19/15/1811.full.html\#ref-list-1

License

Email Alerting

Receive free email alerts when new articles cite this article - sign up in the box at the top

Service

right corner of the article or click here.

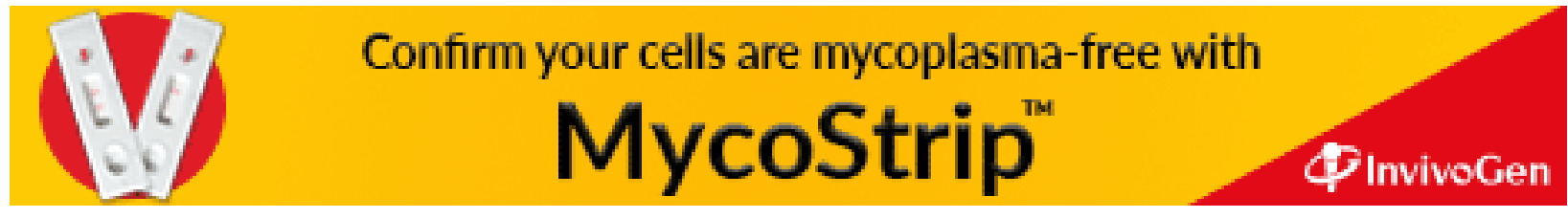

